\title{
The soft X-ray Neupert effect as a proxy for solar energetic particle injection
}

\section{A proof-of-concept physics-based forecasting model}

\author{
Ruhann Steyn $^{1, *}$, Du Toit Strauss ${ }^{1}$, Frederic Effenberger ${ }^{2,3}$, and Daniel Pacheco ${ }^{4}$ \\ ${ }^{1}$ Center for Space Research, North-West University, 2531 Potchefstroom, South Africa \\ 2 Helmholtz Centre Potsdam, GFZ German Research Centre for Geosciences, Telegrafenberg, 14473 Potsdam, Germany \\ 3 Bay Area Environmental Research Institute, NASA Research Park, Moffett Field, CA 94035, USA \\ ${ }^{4}$ Institut für Experimentelle und Angewandte Physik, Christian-Albrechts-Universität zu Kiel, 24118 Kiel, Germany
}

Received 24 August 2020 / Accepted 16 November 2020

\begin{abstract}
The acceleration and injection of solar energetic particles (SEPs) near the Sun is one of the major unsolved problems in contemporary SEP transport modeling efforts. Here, we establish a new approach to the injection problem by utilizing a correlation between the soft X-ray thermal emission in solar flares, and their hard X-ray counterpart, the so-called Neupert effect, which is indicative of the presence of non-thermal particles. We show that the resulting injection function, in the initial phase of the flare, is similar to those inferred from inverting the transport problem based on in-situ observations. For few cases, we find early injections with no in-situ correspondence, that can be caused by particles accelerated before there is a magnetic connection between the source and the spacecraft. The method has limitations for long-duration injections, since it is not applicable to the decay phase of the flare where particle trapping might play a role. For a sample of SEP events in 1980, observed with the Helios-1 and IMP8 spacecraft, we show the results of a 2D SEP transport model based on this approach. We discuss that, with this method, a physics-based, real-time operational SEP now-cast model for the heliosphere is feasible.
\end{abstract}

Keywords: solar energetic particles / injection function / Neupert effect / forecasting / nowcasting

\section{Introduction}

Solar energetic particles (SEPs) are a primary topic of research in heliophysics and pose a potential radiation hazard for hardware and astronauts beyond low Earth orbit, in particular for interplanetary travel (see e.g. Posner \& Strauss, 2020), and thus constitute one of the major components of space weather.

The sudden exposure to energetic protons from solar energetic particle (SEP) events beyond the Earth's magnetosphere and en route to and from Mars can, in extreme cases, lead to acute radiation sickness, an impairing, mission-endangering condition for astronauts. Timely warnings of their impending occurrence may significantly reduce radiation exposure by allowing astronauts sufficient time to move to a radiation shelter.

It is thus vital to develop reliable forecasting tools to describe SEP transport throughout the heliosphere and to couple them directly to the observed solar activity. There is, however,

\footnotetext{
*Corresponding author: ruhann. steyn@nwu. ac.za
}

still a lack of detailed, physics-based SEP transport models that are, in principle, capable of running in a real-time operational setting. In particular, the injection of particles into the transport model is a limiting factor. Here, we will address this challenge by introducing a new method to determine the SEP injection based on solar soft X-ray (SXR) activity, using the so-called Neupert effect (Neupert, 1968).

Coronal mass ejections (CMEs) and solar flares are believed to be the primary sources of SEPs (see e.g. Reames, 1999, 2013). While the exact relation between direct flare acceleration and subsequent CME shock acceleration is still unclear, in particular with regard to different particle species (see e.g. Klein \& Dalla, 2017 for a discussion), clear correlations between flare activity signatures and SEP electron observations at 1 AU have been established. Krucker et al. (2007) discuss the correlation between observed hard X-ray (HXR) spectral indices and the in-situ observed electron spectra in impulsive events. This suggests that solar flare radiative signatures can inform the injection of particles into interplanetary space and thus may be used as an injection proxy for subsequent transport modelling. 
Solar flares are eruptive phenomena with observable signatures in a multitude of different wavelength regimes. Most observations trace the thermal properties of the active solar corona, such as in recent history the EUV (extreme ultra-violet) observations by the $\mathrm{SOHO}$ (Solar and Heliospheric Observatory) and SDO (Solar Dynamics Observatory) missions. For particle injection, however, non-thermal signatures are of particular interest. These are primarily the radio and HXR emissions from non-thermal electrons in the flaring corona. The latter have been extensively studied with the imaging spectroscopy mission RHESSI (Lin et al., 2002), which was recently decommissioned. Routine observations of solar HXR emissions for space weather applications are generally not available, but the SXR observations from the GOES (Geostationary Operational Environmental Satellite) satellites (Garcia, 1994) have a very high duty cycle. By using relations between the SXR and HXR flux, we can thus utilize a reliable and ubiquitously available data source that carries information about non-thermal particles in solar flares.

The original Neupert effect (Neupert, 1968) established a correlation between the the SXR flux and the integral of the microwave flux in solar flares. This is based on energy arguments (Li et al., 1993; Veronig et al., 2002, 2005; Ning \& Cao, 2010), in which the instantaneous flux of non-thermal electrons heats the dense chromosphere and drives evaporation of hot plasma. Thus, the time derivative of the SXR flux, which is an indicator of the thermal plasma and its heating, should correlate with the HXR flux, which in turn is an indicator of non-thermal particles. Figure 1 illustrates the radiation and particle emissions related to a solar flare and the Neupert effect. The electrons in the figure do not refer to electrons that may be accelerated in an associated shock. Additional processes, such as heat conduction and particle escape processes can alter this strict relationship (see e.g. the discussion in Liu et al., 2006). However, Effenberger et al. (2017) found that even in a larger sample of partially occulted flares with pure loop-top emission, the SXR-HXR correlation holds surprisingly well. Furthermore, it has to be noted that the relation between flare particles producing non-thermal emission near the footpoints or even in the loop-top acceleration region and particles escaping as SEPs may involve additional processes and requires further study. Encouragingly, observations do seem to show that the derived SEP release time for impulsive events often coincide well with hard X-ray emission (Tylka et al., 2003). In general, the SXR-HXR relationship can be a powerful proxy for SEP transport models aimed at an operational setting.

Currently, a number of SEP (proton) forecasting models and/or systems exist that uses empirical relationships between e.g. properties of the observed soft X-ray flux and SEP peak intensities (e.g. Núñez, 2011, 2015), which are established from observational correlations (see e.g. Kahler \& Ling, 2018a, 2018b). Other models aim to also include remote-sensing information of the eruptive active region or the corresponding coronal mass ejection (e.g. Balch, 2008; Laurenza et al., 2009, 2018; Alberti et al., 2017; St. Cyr et al., 2017; Richardson et al., 2018), while Posner (2007) correlates the rise of SEP electron intensities with the proton flux observed later on. A detailed list of these forecasting approaches and systems was recently presented by Aminalragia-Giamini et al. (2020). A physics-based SEP forecasting model is presented by Marsh et al. (2015), although this model cannot be used in real-time

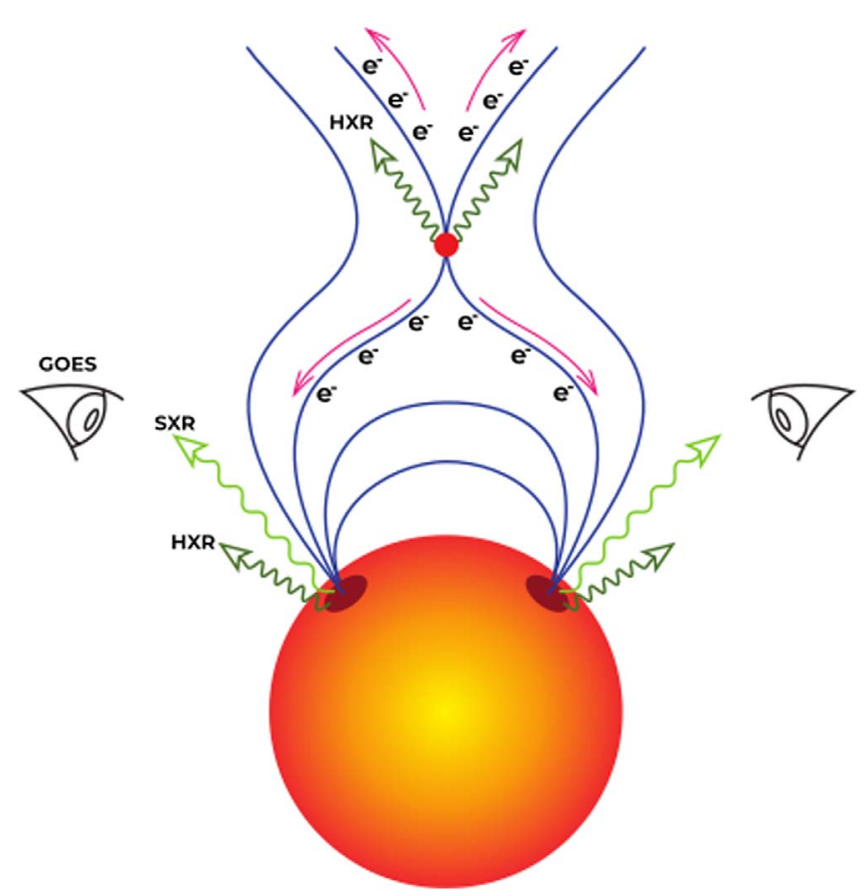

Fig. 1. Illustration of the Neupert effect showing the causal relationship between SXR and HXR emission. Electrons are accelerated near the reconnection region (red dot) whereafter they propagate back to the Sun as well as radially outward. The footpoints and the reconnection region become sources of SXR and HXR.

but is rather based on a number of pre-computed simulations assuming different sources and/or transport parameters. Essentially, two aspects make real-time SEP forecasting with physics-based models operationally ineffective: The first is the appropriate (inner) boundary condition (i.e. the time-intensity profile of SEPs accelerated in the corona) that needs to be specified in any physics-based model. The second is the computational time needed to make a prediction, which, for some models, may take several hours on a dedicated computer cluster. In this paper we address both issues: (i) We propose the use of the Neupert effect as a proxy for particle acceleration in the corona. (ii) Adopting a 2D spatial geometry for the simulation results, and neglecting adiabatic energy losses, computation time is significantly decreased, and SEP predictions can be simulated on a normal desktop PC. The simulations shown here took $1 \mathrm{~h}$ to complete $16 \mathrm{~h}$ of simulation time. Although much more research is required to have an operational model, we combine these ideas into a proof-of-concept model that is used to now-cast the SEP electron distribution in the inner heliosphere.

\section{Comparison with previous injection estimates}

The four consecutive SEP events originating from NOAA Active Region \#2470 on 28 May 1980 have been studied extensively in the past due to good satellite coverage at the time (Gaizauskas, 1982). These SEP events were observed by the GOES-3, Helios-1, and the IMP-8 satellites. All four events 

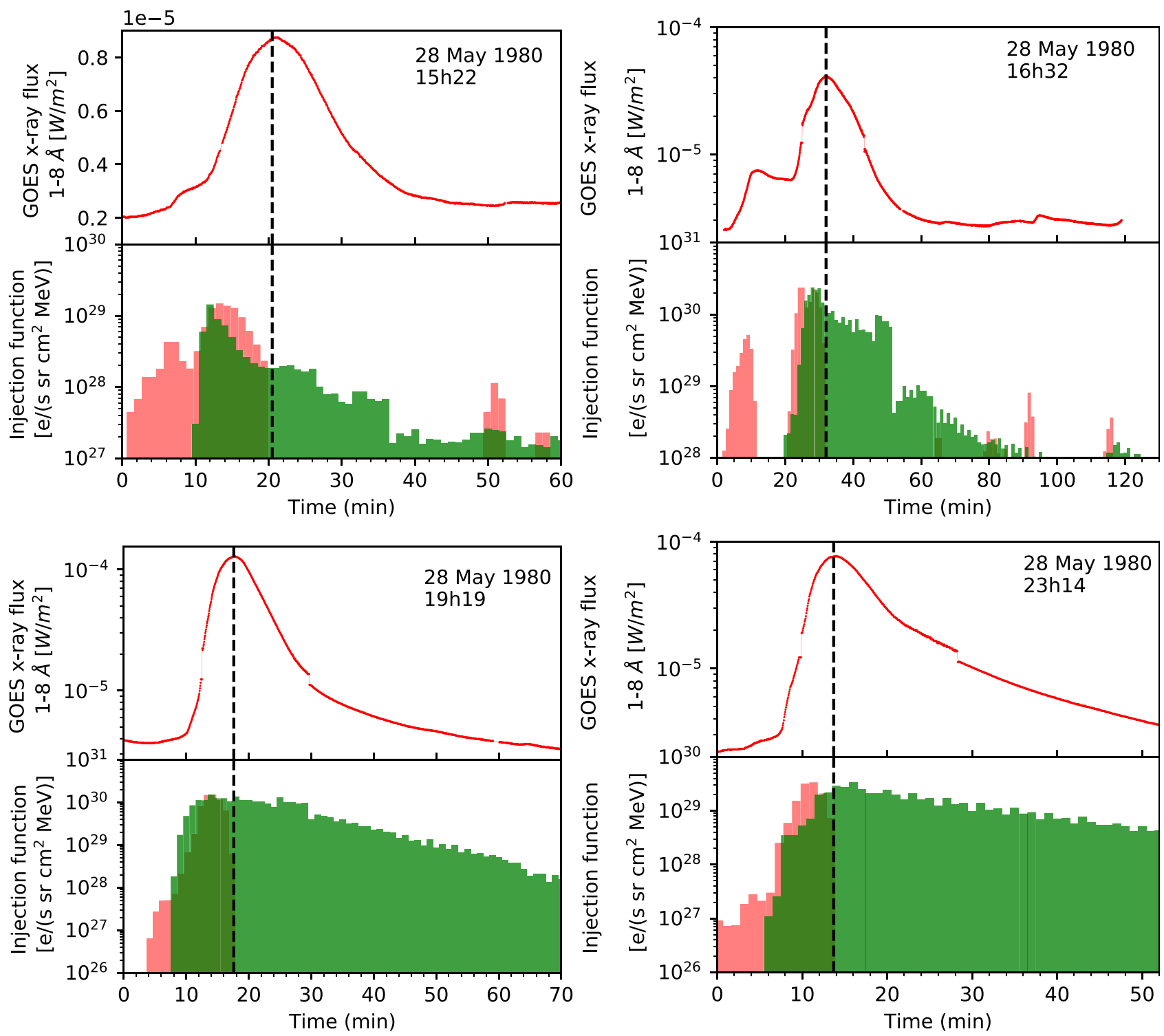

Fig. 2. GOES-3 soft X-ray flux (top panels), the time derivative of the soft X-ray flux (red bars), and the results of Pacheco et al. (2019) (green bars) of the four consecutive SEP events of 28 May 1980. The time in each plot is solar time.

were well defined and occurred in a relatively short period of time $(\sim 10 \mathrm{~h})$. There are no in-situ data for the injection function and therefore it can only be inferred. Agueda \& Lario (2016) uses the observed pitch-angle distributions (PADs) of the SEP events of 28 May 1980 fitted with an exponential function to extract the transport conditions and the release time history of electrons at the Sun by using an inversion method that utilises the results of 1D particle transport model (Agueda et al., 2008). Although Agueda \& Lario (2016) cannot be used for forecasting, it still presents an opportunity to compare with the results of the Neupert effect for these SEP events.

Pacheco et al. (2019) studied a sample of 15 electron events observed by the Helios mission, with the 4 events observed on 28 May 1980 among them. They used the interplanetary transport model by Agueda (2008) to compute the Green's functions of different interplanetary transport scenarios, i.e. different values of the electron radial mean free path, assuming a static solar source at two solar radii and a constant solar wind speed. Then, they performed a full inversion procedure to infer the characteristics of the particle transport for each event, meaning they used the sectored intensities to find the injection profile at the Sun and the value of the radial mean free path that best fitted the observations. The novelty of their approach, as the inversion method has been used on multiple occasions (e.g. Agueda et al., 2009, 2014; Pacheco et al., 2017), was that for the first time, they took into account both the energetic response of the instrument, E6, as well as the angular response of its sectors. An extensive study of this inversion method can be found in Pacheco (2019). Unfortunately, the inversion method may present some inconveniences when fitting data strongly affected by noise or data gaps. This data issues can produce, 
on occasion, an overestimation of the injections around data gaps, or spurious injections when the program attempts to fit data points strongly affected by noise. For that reason, different 4-point smooths has been applied to the injection results from Pacheco et al. (2019), but only those for which it produced a significant improvement have been used in this study: for the four events on 28 May 1980, the smooth was applied only to the injections corresponding to decay phase of the events; for the event on 10 June 1981, the smooth has been applied to the whole injection profile. For this study, the authors selected those events showing no signatures of interplanetary shocks either between the Sun and the spacecraft or beyond it, that could have distorted the interplanetary magnetic field, strongly affecting the path length of the particles. This condition also discards the scenario of having shock-driven acceleration for the observed electrons, which is not considered in their model. The fact that the inversions, in general, matched the event profiles is a good indicator that the selection was accurate, and the electrons are accelerated at the Sun.

The GOES-3 SXR flux of the 28 May 1980 SEP events are shown in the top of each panel in Figure 2, with the time of peak intensity indicated with a vertical dashed line. The time-derivative of the GOES-3 SXR flux (disregarding, of course, negative values), which according to the Neupert effect is indicative of the HXR flux, is shown as red histograms in the bottom of each panel in Figure 2 and the green histograms are the results of the inversion method reported by Pacheco et al. (2019). Note that the result of the time-derivative was scaled (by eye) to be comparable to that of the SEP inversion algorithm. All four SEP events were classified as extended events, following the definition of Pacheco et al. (2019).

The onset and peak of the injection, as estimated from both approaches, compare reasonably well although minor differences on the onset time can be observed. These discrepancies can be attributed to the distinct nature of the modelling methods that rely on totally different assumptions. We use the Neupert effect to derive the electron release history in the corona from the initial acceleration of the electrons, while Pacheco et al. (2019) use the inversion procedure to obtain the release time of the particles onto open magnetic field lines (with the model started at 2 solar radii) that allow them to travel into interplanetary space. In addition, the estimate using the time derivative is unable to capture the extended tail of the injection as inferred by Pacheco et al. (2019). This could point to an extended trapping of the particles in the flare, before slowly diffusing out of a magnetic trap. A comparison with more events are presented in Appendix B. Interestingly, it seems that the Neupert approach generally compares much better to the inversion method when events show a short injection (see again Pacheco et al., 2019, for the definition of short versus extended).

For some events, e.g. the top right panel of Figure 2, the Neupert approach indicates multiple injections which are not present in the inversion results. This could be due to trapping of electrons in the acceleration region (see e.g. Dresing et al., 2018), or the SXR increase could be due to flaring of another active region that may not have produced in-situ electrons at the spacecraft position used for the inversion. Additional observations, including remote-sensing and radio observations, are needed to disentangle such possible processes.

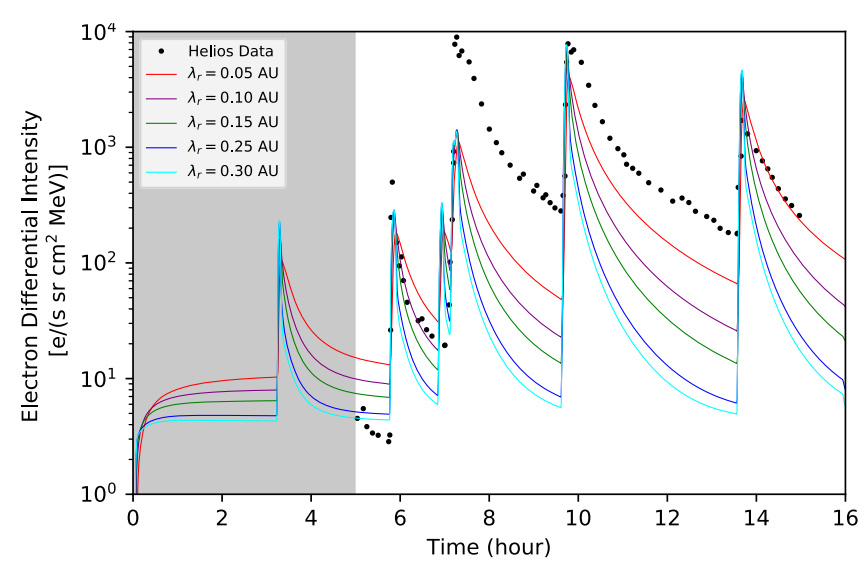

Fig. 3. Simulated temporal evolution of the differential intensity for a range of radial mean free path $\left(\lambda_{r}\right)$ values as indicated in the legend. Note that the model takes $\sim 5 \mathrm{~h}$ to settle into a quasi-steady state (indicated by the grey shading) if initialised with constant SEP injection at the inner boundary.

\section{Simulation results}

Here we make use of the spatially 2D numerical SEP transport model of Strauss \& Fichtner (2015). This model is very well suited for simulations of SEP electron transport, as the adiabatic energy losses and co-rotation effects that are not included in the model should not play a major role for these particles. We also show in Appendix A that results from this model compare very well with those of full 3D simulations, but without being computationally expensive. The present model was used by Strauss et al. (2017) to study SEP electron transport using theoretical motivated transport coefficients. However, for the current application, we choose to use simpler, but well-tested, parametrized versions of the transport coefficients following the approach of Dröge et al. (2014). In this approach, the level of pitch-angle scattering is governed by the effective radial parallel mean-free-path, $\lambda_{r}$, and the level of perpendicular diffusion is governed by a coefficient $a$ (see Eq. 11 of Dröge et al., 2014), where $a=0.13$ seems to be consistent with observations. Following our previous approaches, we specify a Gaussian form, in terms of the azimuthal angle, for the injection function at the inner boundary of the model, with the width of the injection region defined by the broadness of the injection Gaussian function, $\sigma$. Commonly, simulation results will adopt some version of a Reid-Axford (Reid, 1964) temporal injection profile to determine the time dependence of the injected SEP profile. However, here we use the calculations of the injection function, presented in Section 2 and based on the Neupert effect, to determine the temporal evolution at the model's inner boundary.

For the simulation results presented in this section, we adopt the following parameters: $300 \mathrm{keV}$ SEP electrons are simulated for different values of $\lambda_{r}$ ranging between $0.05 \mathrm{AU}$ and 0.30 AU, $a=0.13$ is used in the calculation of the perpendicular diffusion coefficient, and the width of the injected source is varied between $\sigma=5^{\circ}$ (considered a very compact source) and $\sigma=20^{\circ}$ (considered to be a wide injection).

Figure 3 shows simulation results at a radial position of $0.3 \mathrm{AU}$, and good magnetic connectivity to the source, for a 

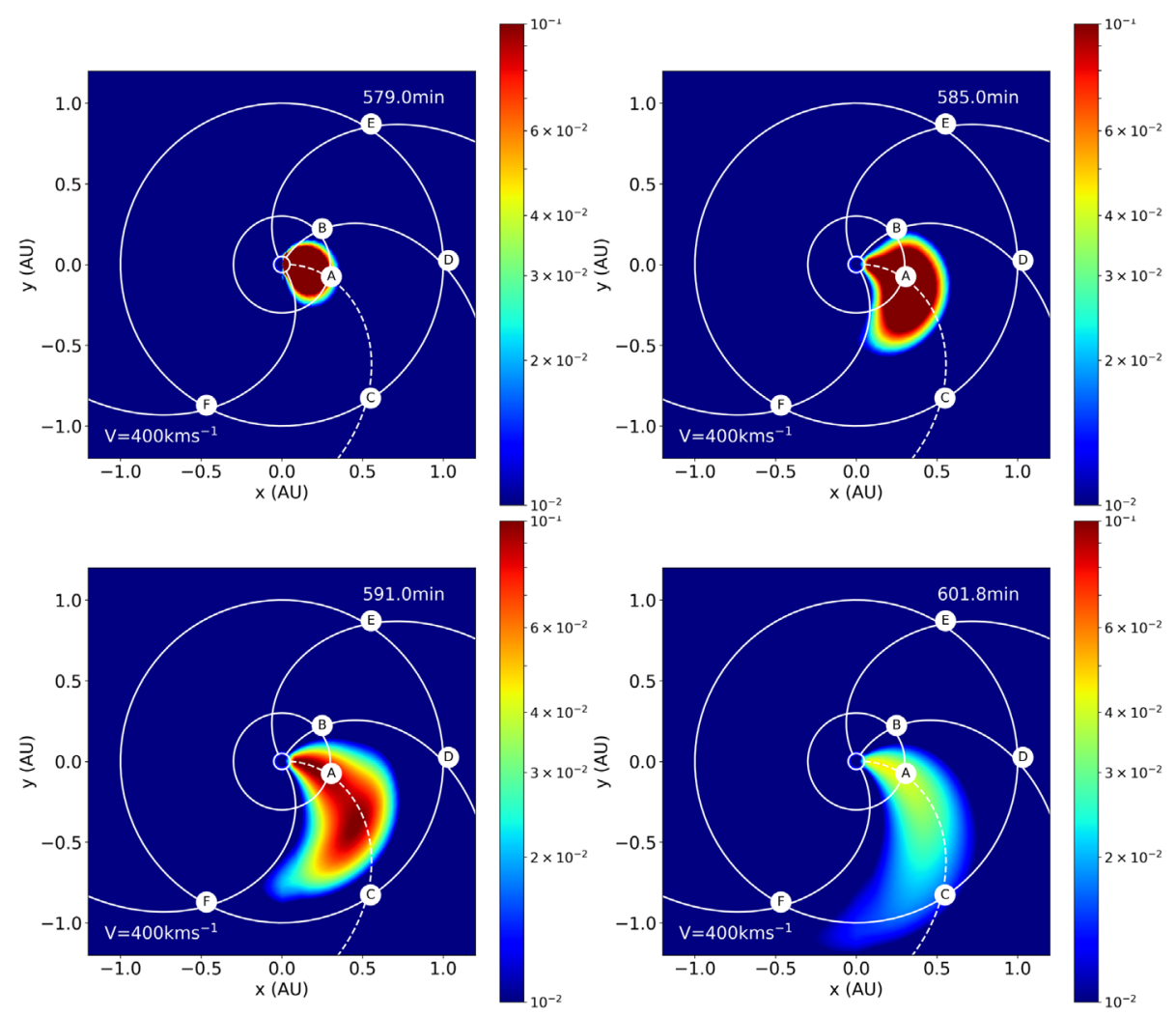

Fig. 4. Contour plots showing the temporal evolution of the omni-directional differential intensity, in the equatorial plane, for the third event of Figure 3. The labelled symbols indicate the position of the six virtual observers assumed in the model. The first frame (579.0 min) shows the peak intensity reaching 0.3 AU while frames 2 and 3, both 6 min apart, show the effect of perpendicular diffusion. The last frame $(\sim 11$ min later) shows the decay phase at $1 \mathrm{AU}$.

wide injection $\left(\sigma=20^{\circ}\right)$ near the Sun. The different lines correspond to different values of $\lambda_{r}$ (indicated in the legend), while the symbols show Helios-1 observations in the energy range of $300-800 \mathrm{keV}$ (Agueda \& Lario, 2016). The simulation results are normalised to the peak of the third SEP event according to Helios-1. The simulated results seem to capture the onset of the event fairly well, while the model underestimates the decay phase, even for the smallest assumed value of $\lambda_{r}=0.05 \mathrm{AU}$. This discrepancy with the measurements is due to the injection function, derived from the Neupert effect, not exhibiting the extended decay phase, as discussed in Section 2. Our proposed approach of using the Neupert effect as a proxy for SEP injection is therefore more applicable in the onset phase of the SEP events.

Note that the model takes $\sim 5 \mathrm{~h}$ to settle into a quasi-steady state (indicated on Fig. 3 by grey shading) if initialised with constant SEP injection at the inner boundary. For this reason we only look at simulation results at later times in order to eliminate any possible numerical artefacts. The simulations presented here were also repeated for $800 \mathrm{keV}$ electrons and the difference was insignificant since electrons from both bins are already relativistic.

Figure 4 shows a collection of contour plots that illustrate the evolution of the omni-directional differential intensities for the third SEP events on 28 May 1980. The dashed white line represents the magnetic field line that is the best magnetically connected field line to the Earth at $1 \mathrm{AU}$, which is indicated as observer C. Observers A and B, at $0.3 \mathrm{AU}$, are located on the best connected magnetic field line and a field line $+45^{\circ}$ westward, respectively. Observers D, E, and F, all located at $1 \mathrm{AU}$, are $+45^{\circ}$ westward, $+90^{\circ}$ westward, and $-90^{\circ}$ eastward, respectively. An injection broadness of $\sigma=20^{\circ}$ and a radial mean free path of $\lambda_{r}=0.25 \mathrm{AU}$ were used in this simulation for the 300 $\mathrm{keV}$ electrons. The 570 min mark indicates the start of the third SEP event (Fig. 4) and the intensity evolves over the next $30 \mathrm{~min}$. The important effect of two dimensional scattering is seen where the SEPs start spreading longitudinally already at a radial distance of $0.3 \mathrm{AU}$ and significantly when the particles reach $1 \mathrm{AU}$.

The top panels of Figure 5 shows the differential SEP intensities, and anisotropy, for an injection broadness of $\sigma=5^{\circ}$ and a radial mean free path of $\lambda_{r}=0.1 \mathrm{AU}$ at the position of the six virtual observers. The bottom panels is for the case of $\sigma=20^{\circ}$ and the same $\lambda_{r}$. For these, and all subsequent simulations, the injection function as inferred by using the Neupert effect is used, while the simulated differential intensity is normalized to observations. The location of the observers are the same as in Figure 4. Helios-1 (at $\sim 0.3 \mathrm{AU}$ and close to best magnetic connection) and $I M P-8$ (at $\sim 1 \mathrm{AU}$ and $\sim 50^{\circ}$ westward of best magnetic connectivity) observations are shown for comparison. 

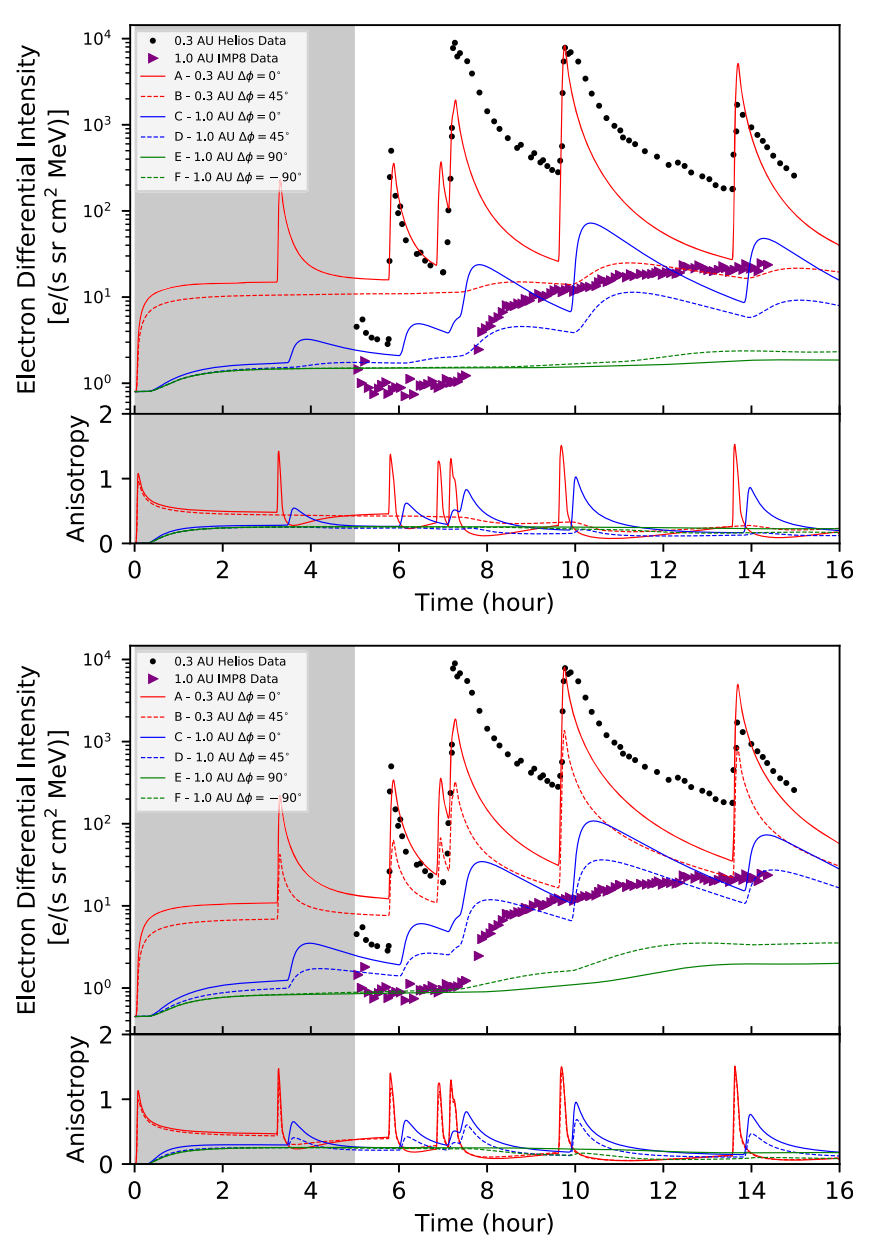

Fig. 5. Simulated omni-directional differential intensities and anisotropies for $\lambda_{r}=0.10 \mathrm{AU}$ and an injection broadness of $\sigma=5^{\circ}$ (top panel) and $\sigma=20^{\circ}$ (bottom panel). Refer to text for a discussion regarding the gray shaded area.

The timing of the peaks from the injections correspond well with the observed intensity peaks from data, specifically the Helios- 1 data at 0.3 AU. The onset phase also corresponds well, with the decay phase again not completely captured by the model. This is, most likely, again related to the extended release of flare particles not captured by the Neupert effect. The simulation results at $1 \mathrm{AU}$ also compare relatively well with the IMP-8 measurements, given that the parameters used here were not tuned to reproduce any observations. The interplay between the effectiveness of perpendicular diffusion and the size of the source region is also clearly visible: Virtual observers magnetically connected to the source directly see the injected time profile, while observers not magnetically connected see a more gradual rise in intensity due to the presence of perpendicular diffusion.

The results presented in Figure 5 therefore illustrate the effects of perpendicular diffusion and magnetic connectivity on the simulation results: In the top panel, observer B is not magnetically connected to the (small/narrow) source, while it is magnetically connected to the (broad/wide) source in the bottom panel. At $1 \mathrm{AU}$ the effect of perpendicular diffusion becomes apparent when comparing e.g. observers $\mathrm{C}$ and $\mathrm{E}$, where SEPs reaching observer E must do so via perpendicular diffusion, and simulations hence show a smooth onset, and associated small anisotropies.

\section{Discussion and conclusions}

The Neupert effect could, potentially, give us the means to estimate, in near real-time, the temporal profile of SEPs injected into the interplanetary medium. In this study, we have compared the injection function, as derived using the Neupert effect, to the inversion results of Pacheco et al. (2019). We find a reasonably good comparison, with the results based on the Neupert effect seemingly capturing the initial particle release effectively. Some deviations, however, between the two approaches exist. Most notably, the results from the Neupert effect does not seem to capture the extended release of SEPs. This extended injection is, most likely, caused by trapping of particles, leading to a slow release (due to diffusion, or leakage of particle out of the acceleration site) of SEPs from the acceleration regions into interplanetary space. For few cases, we find early injections with no in-situ correspondence, that can be caused by particles accelerated before there is a magnetic connection between the source and the spacecraft. Moreover, the Neupert effect cannot give the absolute intensity of SEPs released into interplanetary medium. This can, however, be overcome by comparing (and normalising) calculated SEPs intensities to in-situ observations.

With a proxy for SEP injection readily available, we also illustrate the possibility of developing a real-time, physicsbased, SEP prediction tool. Here, we have used the numerical SEP transport model of Strauss \& Fichtner (2015), driven by an inner boundary condition based on the Neupert injection function results, and compared the resulting SEP intensities to in-situ observations. Results show that this approach captures the SEP onset very efficiently, including the effect of changing magnetic connectivity to different spacecraft. However, our approach is not effective in capturing the decay phase of SEP event. In addition, we believe that a spatially $2 \mathrm{D}$ approach is adequate to capture the essential SEP transport mechanisms, as illustrated in Appendix A.

Here, simulation results are presented for $300 \mathrm{keV}$ electrons, while being compared to the $1-8 \AA$ channel of the GOES satellite. Ideally, the HXR spectrum from an event would be used to look at a specific electron energy corresponding to the available SXR photon band. Since RHESSI has been retired, this is not currently possible. Future studies could include matching electron energies to other GOES photon bands (see e.g. Alcock, 2018). The Spectrometer/Telescope for Imaging X-rays (STIX) instrument on board the Solar Orbiter is a HXR spectrometer and will provide valuable data on the location, spectrum, and energy content of flare-accelerated nonthermal electrons (Krucker et al., 2020). STIX has the potential to provide realtime HXR data which could be used as a proxy for the injection function of the current study.

To move the model presented here into an operational setting will still require additional study, and especially testing to determine its validity in reproducing proton events. Richardson et al. (2014) showed there is a correlation between the GOES X-ray intensity and the $25 \mathrm{MeV}$ proton intensities at Earth. A similar correlation is also found for electrons. These correlations can be used to apply a better normalisation 
and to extend the model for proton events. Posner (2007) also showed that the early electron intensities of an SEP event can be used to forecast the subsequent proton intensities. Using the definition of Halford et al. (2019), the approach described here is currently in Phase 1 of application usability. The choice of interplanetary transport parameters also remains uncertain and a thorough parameter study is yet to be performed. A more comprehensive comparison with existing SEP observations (e.g. the catalogue of Papaioannou et al., 2016), as well as future Parker Solar Probe and Solar Orbiter observations closer to the Sun, could be used in future work to thoroughly examine the validity and application of the Neupert effect as a proxy for the injection function. In the future, ensemble simulations (i.e. simulations with a range of transport and input parameters) will, most likely, be performed to give an indication of the uncertainty related to the simulation results. However, we believe that such a operational model is practical implementable. We illustrate this by having a real-time now-casting version of the model discussed in this paper running at https://fskbhe1.puk.ac.za/spaceweather/ sep_predictor.html. Although this is currently only a first version of a proof-of-concept model, we believe that, with the correct modifications, it could develop into a valuable forecasting resource.

Acknowledgements. FE acknowledges support from NASA grant NNX17AK25G. Additional support from an Alexander von Humboldt group linkage program between Germany and South Africa is appreciated. We thank the International Space Science Institute (ISSI) for hosting our team on "Solar flare acceleration signatures and their connection to solar energetic particles". This work is based on the research supported in part by the National Research Foundation of South Africa (NRF grant number 120847). DP thanks the German Federal Ministry for Economic Affairs and Energy and the German Space Agency (Deutsches Zentrum für Luft- und Raumfahrt, e.V.; DLR) for their unwavering support through grants numbers 50OT0901, 50OT1202, and 50OT1702. Opinions expressed and conclusions arrived at are those of the authors and are not necessarily to be attributed to the NRF. RS acknowledges Marina Herbst for the design of Figure 1. All other figures in this project were created in Matplotlib (Hunter, 2007), a Python 3.6 package. We acknowledge the National Oceanic and Atmospheric Administration database for providing GOES data https://satdat.ngdc.noaa.gov/sem/goes/data/full/fits/xrays/ . The authors also thank the reviewers for their insightful help and suggestions. The editor thanks two anonymous reviewers for their assistance in evaluating this paper.

\section{References}

Agueda N. 2008. Near-relativistic electron events. Monte Carlo simulations of solar injection and interplanetary transport, Ph.D. Thesis, Dep. Astronomia i Meteorologia University of Barcelona, Martí i Franquès 108028 Barcelona, Spain.

Agueda N, Klein K-L, Vilmer N, Rodríguez-Gasén R, Malandraki OE, et al. 2014. Release timescales of solar energetic particles in the low corona. A\&A 570: A5. https://doi.org/10.1051/0004-6361/ 201423549.
Agueda N, Lario D. 2016. Release history and transport parameters of relativistic solar electrons inferred from near-the-sun in situ observations. Astrophys J 829(2): 131. https://doi.org/10.3847/ 0004-637X/829/2/131.

Agueda N, Vainio R, Lario D, Sanahuja B. 2008. Injection and interplanetary transport of near-relativistic electrons: Modeling the impulsive event on 2000 May 1. Astrophys J 675: 1601-1613. https://doi.org/10.1086/527527.

Agueda N, Vainio R, Lario D, Sanahuja B. 2009. The influence of in situ pitch-angle cosine coverage on the derivation of solar energetic particle injection and interplanetary transport conditions. Adv Space Res 44(7): 794-800. https://doi.org/10.1016/j. asr.2009.05.023, URL http://www.sciencedirect.com/science/article/pii/S0273117709003767.

Alberti T, Laurenza M, Cliver EW, Storini M, Consolini G, Lepreti F. 2017. Solar activity from 2006 to 2014 and short-term forecasts of solar proton events using the ESPERTA model. Astrophys J 838(1): 59. https://doi.org/10.3847/1538-4357/aa5cb8.

Alcock B. 2018. Solar electron and radio propagation in the turbulent solar corona, Ph.D. Thesis, College of Science and Engineering, School of Physics and Astronomy, University of Glasgow, Glasgow, Scotland, UK.

Aminalragia-Giamini S, Jiggens P, Anastasiadis A, Sandberg I, Aran A, et al. 2020. Prediction of solar proton event fluence spectra from their peak flux spectra. J Space Weather Space Clim 10: 1 . https://doi.org/10.1051/swsc/2019043.

Balch CC. 2008. Updated verification of the Space Weather Prediction Center's solar energetic particle prediction model. Space Weather 6(1): S01001. https://doi.org/10.1029/2007SW000337.

Dresing N, Gómez-Herrero R, Heber B, Klassen A, Temmer M, Veronig A. 2018. Long-lasting injection of solar energetic electrons into the heliosphere. $A \& A$ 613: A21. https://doi.org/ 10.1051/0004-6361/201731573.

Dröge W, Kartavykh YY, Dresing N, Heber B, Klassen A. 2014. Wide longitudinal distribution of interplanetary electrons following the 7 February 2010 solar event: Observations and transport modeling. J Geophys Res (Space Phys) 119(8): 6074-6094. https://doi.org/10.1002/2014JA019933.

Effenberger F, Rubio da Costa F, Oka M, Saint-Hilaire P, Liu W, Petrosian V, Glesener L, Krucker S. 2017. Hard X-ray emission from partially occulted solar flares: RHESSI observations in two solar cycles. Astrophys J 835(2): 124. https://doi.org/10.3847/ $1538-4357 / 835 / 2 / 124$.

Gaizauskas V. 1982. The relation of solar flares to the evolution and proper motions of magnetic fields. Adv Space Res 2(11): 11-30. https://doi.org/10.1016/0273-1177(82)90175-2.

Garcia HA. 1994. Temperature and emission measure from Goes Soft X-ray measurements. Sol. Phys. 154(2): 275-308. https://doi. org/10.1007/BF00681100.

Halford AJ, Kellerman AC, Garcia-Sage K, Klenzing J, Carter BA, et al. 2019. Application usability levels: a framework for tracking project product progress. J Space Weather Space Clim 9: A34. https://doi.org/10.1051/swsc/2019030.

Hunter JD. 2007. Matplotlib: A 2D graphics environment. Comput Sci Eng 9(3): 90-95. https://doi.org/10.1109/MCSE.2007.55.

Kahler SW, Ling AG. 2018a. Forecasting solar energetic particle (SEP) events with flare X-ray peak ratios. J Space Weather Space Clim 8: A47. https://doi.org/10.1051/swsc/2018033.

Kahler SW, Ling AG. 2018b. Relating solar energetic particle event fluences to peak intensities. Sol Phys 293(2): 30. https://doi.org/ 10.1007/s11207-018-1249-x. 
Klein K-L, Dalla S. 2017. Acceleration and propagation of solar energetic particles. Space Sci Rev 212(3-4): 1107-1136. https://doi.org/10.1007/s11214-017-0382-4.

Krucker S, Hurford GJ, Grimm O, Kögl S, Gröbelbauer HP, et al. 2020. The spectrometer/telescope for imaging X-rays (STIX). A\&A 642: A15. https://doi.org/10.1051/0004-6361/201937362.

Krucker S, Kontar EP, Christe S, Lin RP. 2007. Solar flare electron spectra at the Sun and near the Earth. Astrophys J Lett 663(2): L109-L112. https://doi.org/10.1086/519373.

Laurenza M, Alberti T, Cliver EW. 2018. A short-term ESPERTAbased forecast tool for moderate-to-extreme solar proton events. Astrophys J 857(2): 107. https://doi.org/10.3847/1538-4357/aab712.

Laurenza M, Cliver EW, Hewitt J, Storini M, Ling AG, Balch CC, Kaiser ML. 2009. A technique for short-term warning of solar energetic particle events based on flare location, flare size, and evidence of particle escape. Space Weather 7(4): S04008. https://doi.org/10.1029/2007SW000379.

Li P, Emslie AG, Mariska JT. 1993. Implications of the soft X-ray versus hard X-ray temporal relationship in solar flares. Astrophys $J$ 417: 313. https://doi.org/10.1086/173314.

Lin RP, Dennis BR, Hurford GJ, Smith DM, Zehnder A, et al. 2002. The Reuven Ramaty High-Energy Solar Spectroscopic Imager (RHESSI). Sol Phys 210(1): 3-32. https://doi.org/10.1023/ A:1022428818870.

Liu W, Liu S, Jiang YW, Petrosian V. 2006. RHESSI observation of chromospheric evaporation. Astrophys J 649(2): 1124-1139. https://doi.org/10.1086/506268.

Marsh MS, Dalla S, Dierckxsens M, Laitinen T, Crosby NB. 2015. SPARX: A modeling system for solar energetic particle radiation space weather forecasting. Space Weather 13(6): 386-394. https://doi.org/10.1002/2014SW001120.

Neupert WM. 1968. Comparison of solar X-ray line emission with microwave emission during flares. Astrophys J Lett 153: L59. https://doi.org/10.1086/180220.

Ning Z, Cao W. 2010. Investigation of chromospheric evaporation in a Neupert-type solar flare. Astrophys J 717(2): 1232-1242. https://doi.org/10.1088/0004-637X/717/2/1232.

Núñez M. 2011. Predicting solar energetic proton events $(\mathrm{E}>10$ MeV). Space Weather 9(7): 07003. https://doi.org/10.1029/ 2010SW000640.

Núñez M. 2015. Real-time prediction of the occurrence and intensity of the first hours of $>100 \mathrm{MeV}$ solar energetic proton events. Space Weather 13(11): 807-819. https://doi.org/10.1002/2015SW001256.

Pacheco D. 2019. Analysis and modelling of the solar energetic particle radiation environment in the inner heliosphere in preparation for solar orbiter, Ph.D. Thesis, Dep. Física Quàtica i Astrofísica, Universitat de Barcelona, Barcelona, Spain. URL http://hdl.handle. net/10803/667033.

Pacheco D, Agueda N, Aran A, Heber B, Lario D. 2019. Full inversion of solar relativistic electron events measured by the Helios spacecraft. A\&A 624: A3. https://doi.org/10.1051/0004-6361/ 201834520.

Pacheco D, Agueda N, Gómez-Herrero R, Aran A. 2017. Interplanetary transport of solar near-relativistic electrons on 2014 August 1 over a narrow range of heliolongitudes. J Space Weather Space Clim 7(27): A30. https://doi.org/10.1051/swsc/2017029.
Papaioannou A, Sandberg I, Anastasiadis A, Kouloumvakos A, Georgoulis MK, Tziotziou K, Tsiropoula G, Jiggens P, Hilgers A. 2016. Solar flares, coronal mass ejections and solar energetic particle event characteristics. J Space Weather Space Clim 6: A42. https://doi.org/10.1051/swsc/2016035.

Posner A. 2007. Up to 1-hour forecasting of radiation hazards from solar energetic ion events with relativistic electrons. Space Weather 5(5): 05001. https://doi.org/10.1029/2006SW000268.

Posner A, Strauss RD. 2020. Warning time analysis From SEP simulations of a two-tier REleASE system applied to Mars exploration. Space Weather 18(4): e02354. https://doi.org/ 10.1029/2019SW002354.

Reames DV. 1999. Particle acceleration at the Sun and in the heliosphere. Space Sci Rev 90: 413-491. https://doi.org/10.1023/ A: 1005105831781 .

Reames DV. 2013. The two sources of solar energetic particles. Space Sci Rev 175(1-4): 53-92. https://doi.org/10.1007/s11214013-9958-9.

Reid GC. 1964. A diffusive model for the initial phase of a solar proton event. J Geophys Res 69(13): 2659-2667. https://doi.org/ 10.1029/JZ069i013p02659.

Richardson IG, Mays ML, Thompson BJ. 2018. Prediction of solar energetic particle event peak proton intensity using a simple algorithm based on CME speed and direction and observations of associated solar phenomena. Space Weather 16(11): 1862-1881. https://doi.org/10.1029/2018SW002032.

Richardson IG, von Rosenvinge TT, Cane HV, Christian ER, Cohen CMS, Labrador AW, Leske RA, Mewaldt RA, Wiedenbeck ME, Stone EC. 2014. >25 MeV proton events observed by the high energy telescopes on the STEREO A and B spacecraft and/or at Earth during the first $\sim$ seven years of the STEREO mission. Sol Phys 289 (8): 3059-3107. https://doi.org/10.1007/s11207-014-0524-8.

St. Cyr OC, Posner A, Burkepile JT. 2017. Solar energetic particle warnings from a coronagraph. Space Weather 15(1): 240-257. https://doi.org/10.1002/2016SW001545.

Strauss RD, Dresing N, Engelbrecht NE. 2017. Perpendicular diffusion of solar energetic particles: Model results and implications for electrons. Astrophys $J$ 837(1): 43. https://doi.org/10.3847/ 1538-4357/aa5df5.

Strauss RD, Fichtner H. 2015. On aspects pertaining to the perpendicular diffusion of solar energetic particles. Astrophys $J$ 801(1): 29. https://doi.org/10.1088/0004-637X/801/1/29.

Tylka AJ, Cohen CMS, Dietrich WF, Krucker S, McGuire RE, Mewaldt RA, Ng CK, Reames DV, Share GH. 2003. Onsets and release times in solar particle events. In: International Cosmic Ray Conference, Vol. 6 of International Cosmic Ray Conference, 3305.

Veronig A, Vršnak B, Dennis BR, Temmer M, Hanslmeier A, Magdalenić J. 2002. Investigation of the Neupert effect in solar flares. I. Statistical properties and the evaporation model. $A \& A$ 392: 699-712. https://doi.org/10.1051/0004-6361:20020947.

Veronig AM, Brown JC, Dennis BR, Schwartz RA, Sui L, Tolbert AK. 2005. Physics of the Neupert effect: Estimates of the effects of source energy, mass transport, and geometry using RHESSI and GOES data. Astrophys J 621(1): 482-497. https://doi.org/10.1086/ 427274. 


\section{Appendix A}

\section{Comparing 2D and 3D simulations}

In order to show that a 2D approach is a reasonable approximation, and therefore includes the most important physics processes necessary to fully describe SEP transport, we compare our 2D simulation results (using a finite difference approach) to the 3D simulations (using a stochastic differential equation approach) of Dröge et al. (2014). Specifically, we reproduce the widespread 7 February 2010 event observed simultaneously by STEREO A, $B$, and the $A C E$ satellites, using exactly the same satellite positions and transport parameters as Dröge et al. (2014); we use $\lambda_{r}=0.08 \mathrm{AU}, a=0.13$, and an injection broadness of $\sigma=26^{\circ}$. While, in the 2D approach, we inject particles only in the equatorial plane, Dröge et al. (2014) uses the precise position of the active region, located $21^{\circ}$ above the equatorial plane. A comparison between the $2 \mathrm{D}$ and $3 \mathrm{D}$ modelling approaches is shown in Figure A.1, with the differential intensity compared in the top panel, and the resulting particle anisotropies in the bottom panel. The simulation results compare very well, with the $2 \mathrm{D}$ model having a slightly quicker onset, being, of course, closer to the source in the reduced 2D geometry. The very good comparison lasts for the first $\sim 10 \mathrm{~h}$ of the event, after which it starts breaking down due to the effect of co-rotation and energy losses not being taken into account in the 2D model. Even with these slight deviations at later times, we are confident that our 2D modelling approach is valid when describing SEP events, even if the active regions may be located significantly away from the equatorial plane.
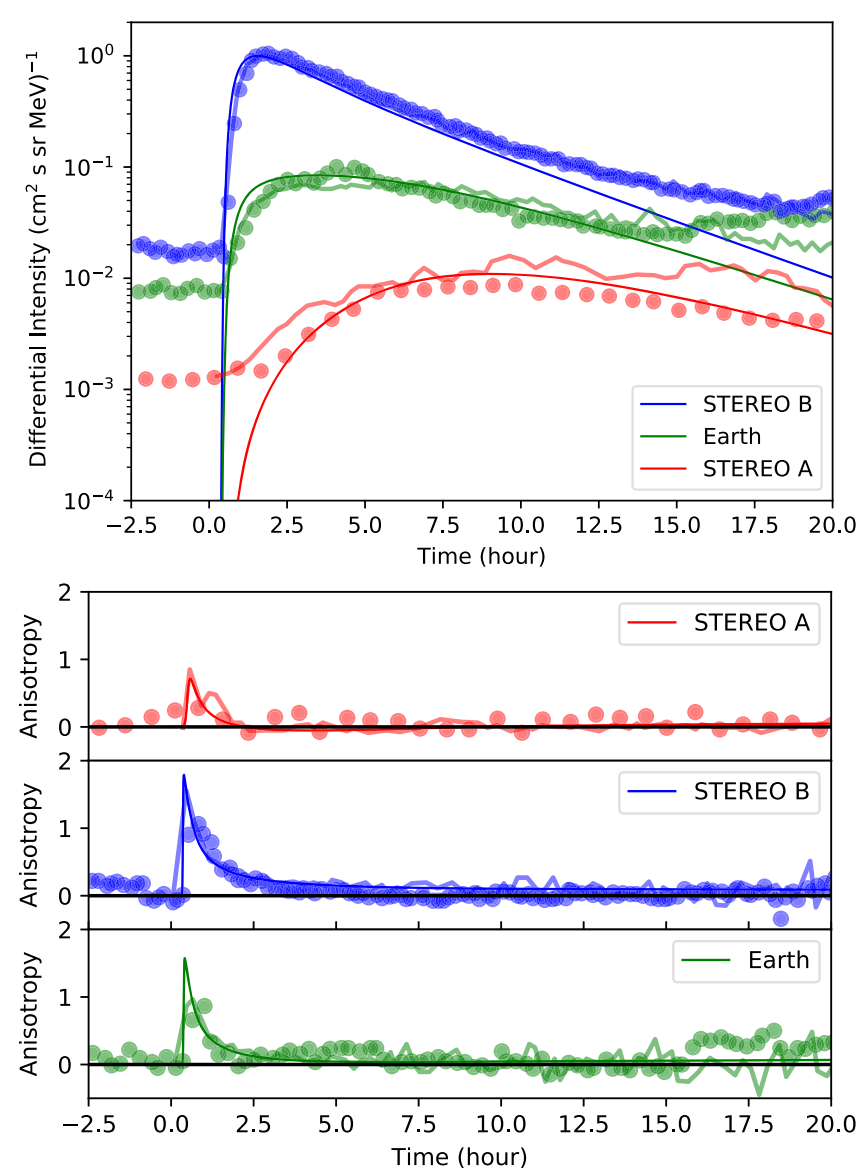

Fig. A.1. The top panel shows a comparison between STEREO A (red), STEREO B (blue), and ACE (Earth, green) observations (data points), modelling solutions from a $3 \mathrm{D}$ stochastic differential equation (SDE, thick curves) model, and a 2D model employing a finite difference (FD, thin lines) approach. The top panel compares the differential intensity and the bottom panel the resulting particle anisotropy. 


\section{Appendix B}

Additional events from Pacheco et al. (2019)

Here we compare the time-derivative approach of calculating the injection function, using the Neupert effect, to the inversion technique of Pacheco et al. (2019) for all events considered in the latter publication. Figure B.1 compares the so-called short duration events, and Figure B.2 the extended events. The Neupert results are again normalized to the inversion results by eye.
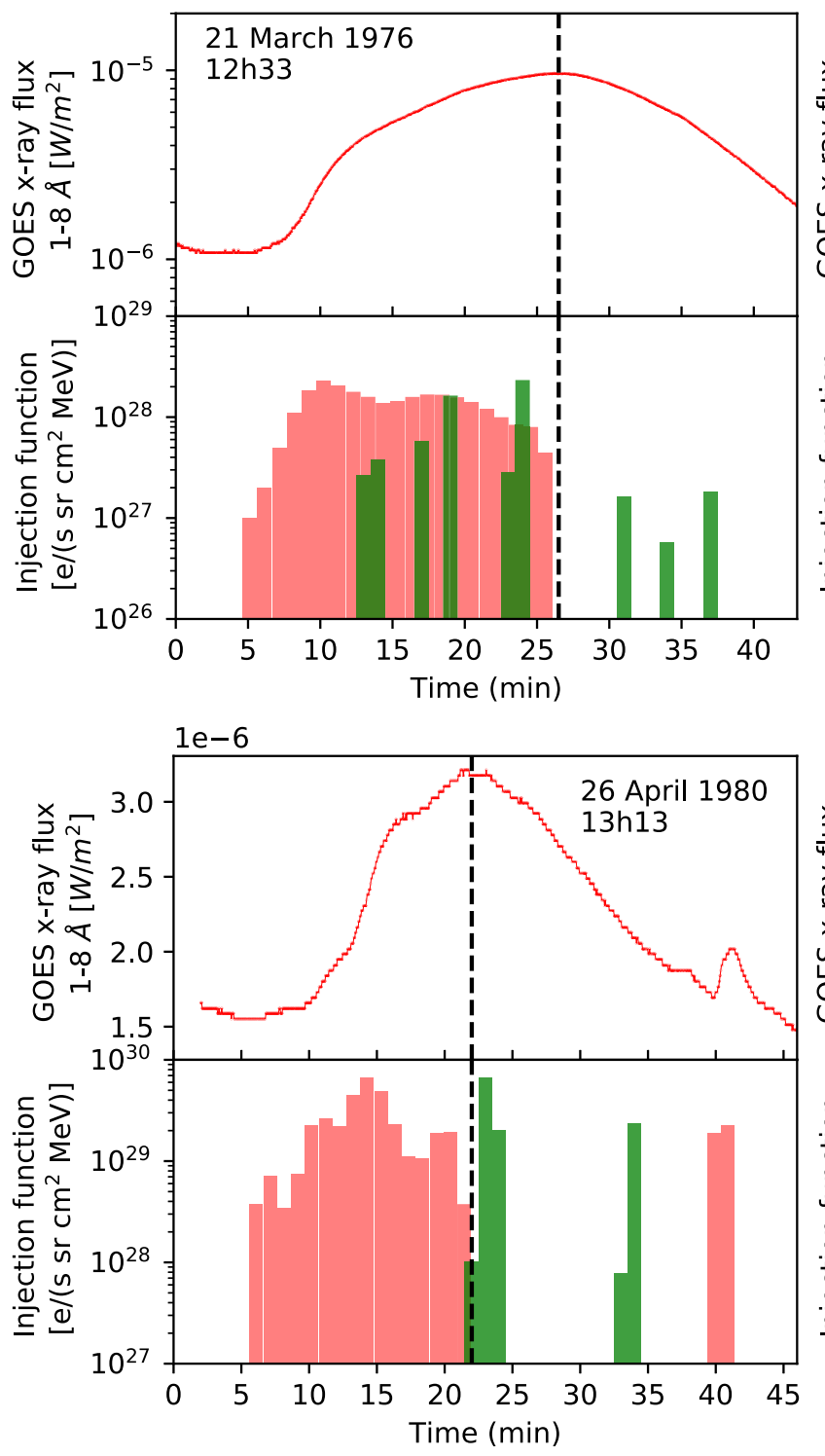

For both the short and extended events, the time derivative of the GOES-3 X-ray flux is consistently earlier than the results of the full inversion method except for the event of 5 April 1980, although this could be due to insufficient temporal resolution in the inversion results. The amount of time with which the inversion method trails behind the Neupert effect varies between events, but could hint at different levels of particle trapping near the acceleration site. The decay phases represented by the two methods differ significantly, and, as alluded to earlier, the Neupert effect does not capture the extended decay/release of particles.
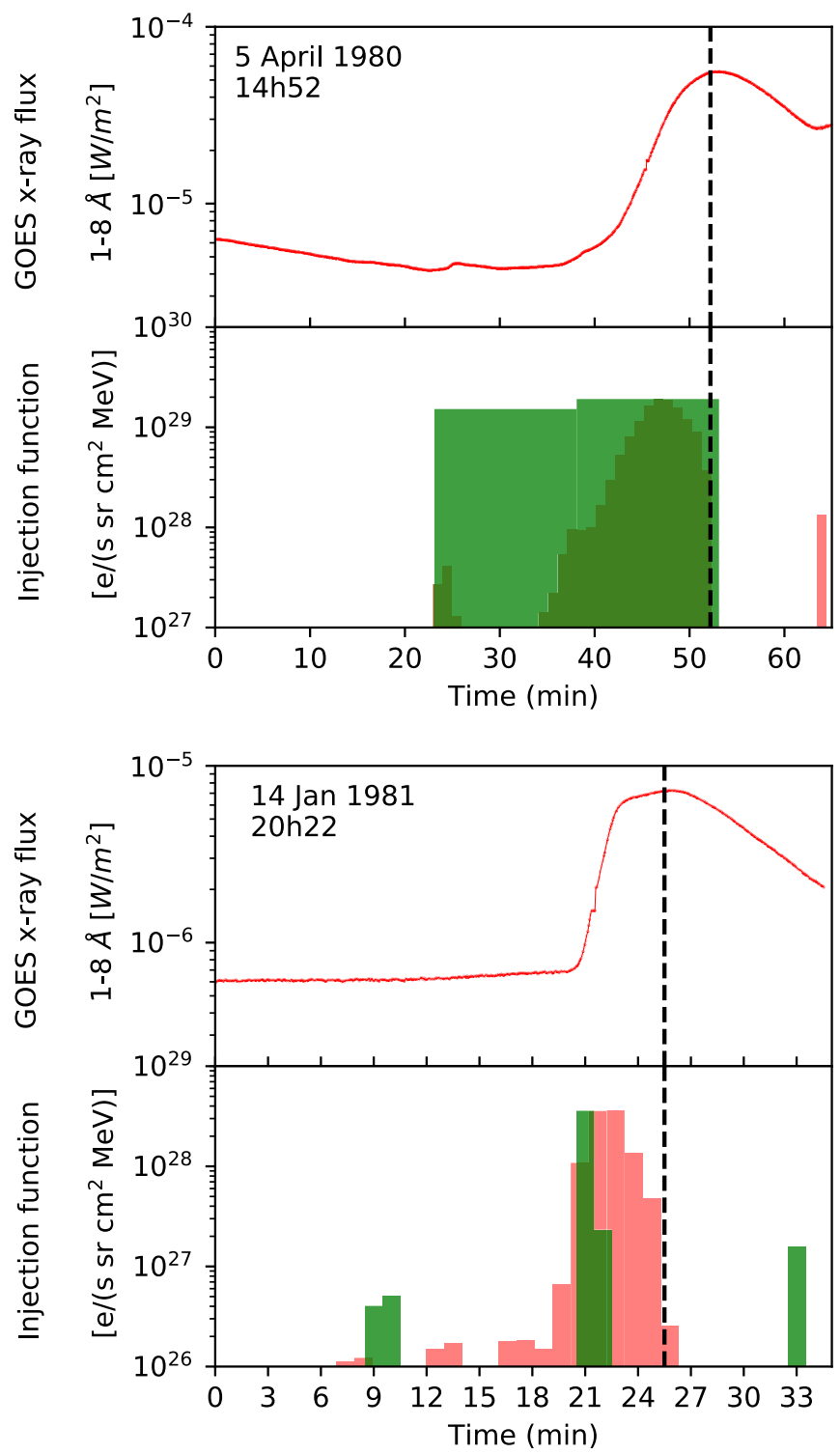

Fig. B.1. GOES-3 SXR flux (top panels), the time derivative of the SXR flux (red bars), and the results of Pacheco et al. (2019) (green bars) for short duration events. The time in each plot is solar time. 

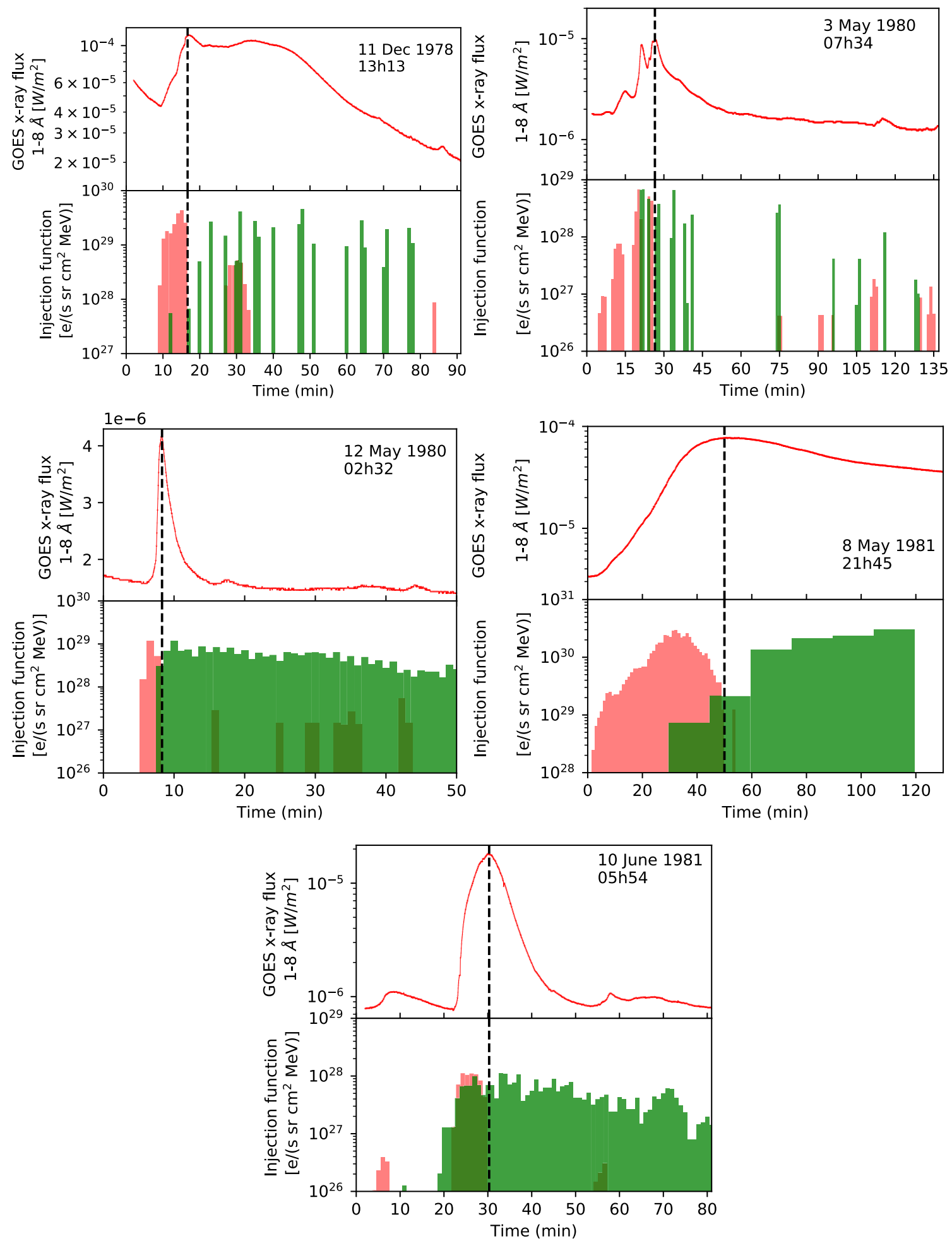

Fig. B.2. Similar to Figure B.1, for extended events.

Cite this article as: Steyn R, Strauss DT, Effenberger F \& Pacheco D 2020. The soft X-ray Neupert effect as a proxy for solar energetic particle injection. J. Space Weather Space Clim. 10, 64. https://doi.org/10.1051/swsc/2020067. 